\title{
The New Detection Technique of Phase-Shift Keyed Signals
}

\author{
Vladimir P. Litvinenko ${ }^{1}$, Yulia V. Litvinenko ${ }^{1}$, Boris V. Matveev ${ }^{1}$, Oleg V. Chernoyarov ${ }^{2, *}$ and Alexander A. \\ Makarov $^{2}$ \\ ${ }^{1}$ Russia, 394026, Voronezh, Moscow Avenue, 14, Voronezh State Technical University, Dept. of Radio Engineering \\ ${ }^{2}$ Russia, 111250, Moscow, Krasnokazarmennaya st, 14, National Research Institute "Moscow Power Engineering Institute", Dept. \\ of Radio Engineering Devices and Antenna Systems \\ ${ }^{*}$ Corresponding author
}

\begin{abstract}
We are to introduce the phase-shift keyed signal detector with a relatively simple construction, the one that provides the high-noise immunity. We are demonstrating that the specified detector can extract the phase-shift keyed signals when the signal-to-noise ratio is much less than its working value in the receiver highway. We have determined the expressions for the false-alarm and missing probabilities if the binary phase-shift keyed signal is detected, and we consider the influence of detector parameters on detection performances. The obtained results are corroborated by statistical simulation. We are also to illustrate that the suggested detector can be practically implemented on the base of both analog and digital devices.
\end{abstract}

Keywords-phase-shift keying; signal detection; noise interference; false-alarm and missing probabilities; noise immunity

\section{INTRODUCTION}

The need to detect the phase-shift keyed (PSK) signals arises in various radio systems, for example, such as detection of radio channel activity in multichannel communications using PSK signals, control of PSK signal receiver, quality control of PSK communication channel, radio reconnaissance of communications with PSK signals, control of digital PSK modems for wire and radio communication channel, et al.

In order to form the reference signal for the $m$-fold PSK signal coherent demodulator the method based on receive signal frequency multiplication, narrow-band filtration and then frequency division in $m$ times is used [1-3], as the signal phase-shift keying is removed by multiplying the frequency. In practice we have $m=2$ usually, and the quadratic transformation of the input signal is applied for the frequency doubling.

\section{PHASE-SHIFT KEYED SIGNAL DETECTOR}

When PSK signal has been detected it is necessary to execute the frequency multiplication, to extract the transform and then to compare this transform to the chosen threshold for a corresponding decision. The detection threshold may be prudent to fix according to the noise rating at the detector input. This technique is implemented in the PSK signal detector [4] the block diagram of which is shown in Figure 1
The input PSK signal $s_{P S K}(t)$ mixed with interferences (for example, noise from the output of the intermediate frequency section of the receiver) is being routed to the input filter $(F)$ the bandwidth $F_{F}$ of which is equal to the signal spectrum width $F_{S}$. For the binary phase shift keying $(m=2)$ we can write down

$$
s_{P S K}(t)=S \cos \left(2 \pi f_{0} t+\psi+a \pi / 2\right)_{1}
$$

Here $S$ is the amplitude, $\psi$ is the initial phase, $f_{0}$ is the carrier frequency, and $a= \pm 1$ is the binary information symbol. The binary symbol duration is $\tau=T_{0} N$, where $T_{0}=1 / f_{0}$ is the carrier cycle and $N$ is the number of periods within one information element. As an example, we presuppose that $S=1, f_{0}=10 \mathrm{MHz}, T_{0}=0.1 \mu \mathrm{s}, N=64$.

Then the symbol duration is equal to $\tau=6.4 \mu$ s and PSK signal spectrum width can be found as $F_{s}=2 / \tau=312.5 \mathrm{kHz}$.

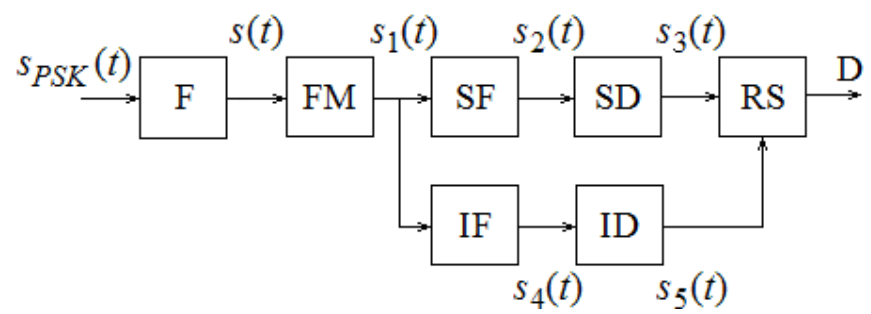

FIGURE I. BLOCK DIAGRAM OF THE PSK DETECTOR

At the output of the filter $F$ with the bandpass $F_{F}=300 \mathrm{kHz}$ we have the PSK signal $s(t)$ with random values of information symbols. In Figure 2 a the example of such waveform diagram is shown, and in Figure $2 \mathrm{~b}$ amplitude spectrum $G(f)$ of this signal is presented together with input filter F skirt plotted by a dotted line.

The frequency multiplier (FM) in Figure 1 is the nonlinear (quadratic) function generator for the signal $s(t)$. The frequency doubling is implemented by a square-law unit on the base of the multiplier. Then for the ideal input signal (1) the FM response is 


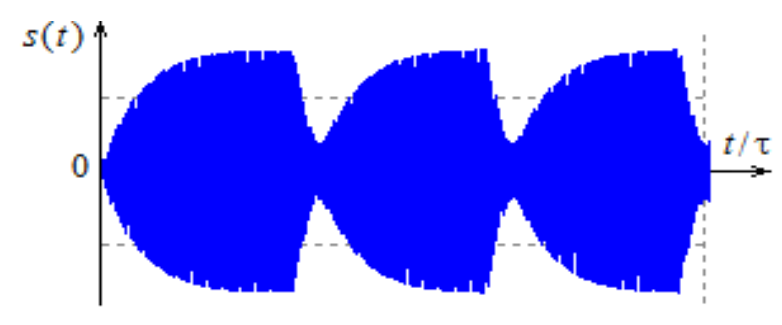

(a)

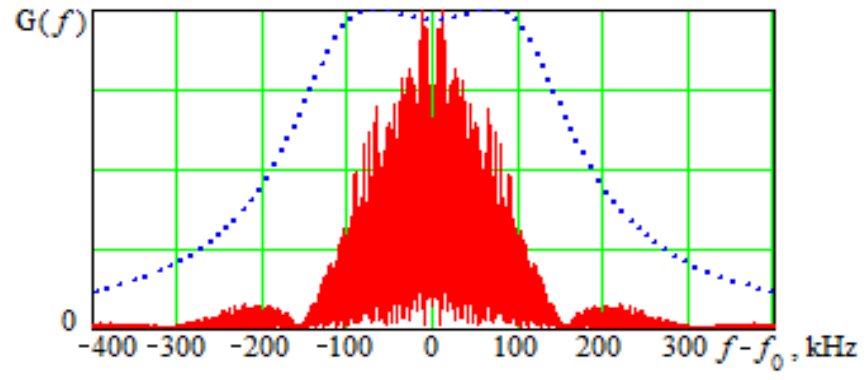

(b)

FIGURE II. PSK SIGNAL AT THE NARROW-BAND FILTER OUTPUT (A) AND PSK SIGNAL SPECTRUM (B)

$$
s_{1}(t)=\left[s_{P S K}(t)\right]^{2}=0.5+0.5 S^{2} \cos \left(4 \pi f_{0} t+2 \psi t\right)
$$

As follows from Eq. (2), FM response does not depend on a modulating signal and it is harmonic oscillation. The amplitude of real PSK signals (Figure 2a) at the FM input changes in time. Therefore, the output FM signal has pulse character, with changing the amplitude. Its spectrum $G_{1}(f)$ is shown in Figure 3. As can be seen, the frequency multiplication transforms the spread spectrum of the input PSK signal to the narrow-band spectrum with the pronounced harmonic components on a frequency of $2 f_{0}$.

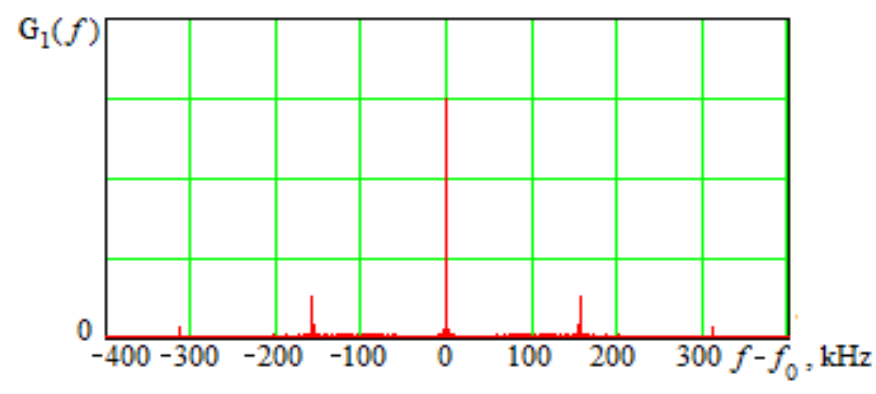

FIGURE III. PSK SIGNAL SPECTRUM AT THE FREQUENCY MULTIPLIER OUTPUT

The FM response is passed to the signal narrow-band filter (designated in Figure 1 a as SF) tuned to a frequency $2 f_{0}$. For example, with the bandpass of $300 \mathrm{~Hz}$ SF skirt is presented in Figure $4 \mathrm{a}$ by the dotted line. In the same place the spectrum of the component of the FM response in the neighborhood of frequency $2 f_{0}$ is drawn by the bars. Its sidetones are caused by fluctuations of the amplitude of a signal at the FM input. In
Figure $4 \mathrm{~b}$ the SF response is shown. The less SF bandpass, the output signal amplitude rises more slowly and the detector noise immunity is better.

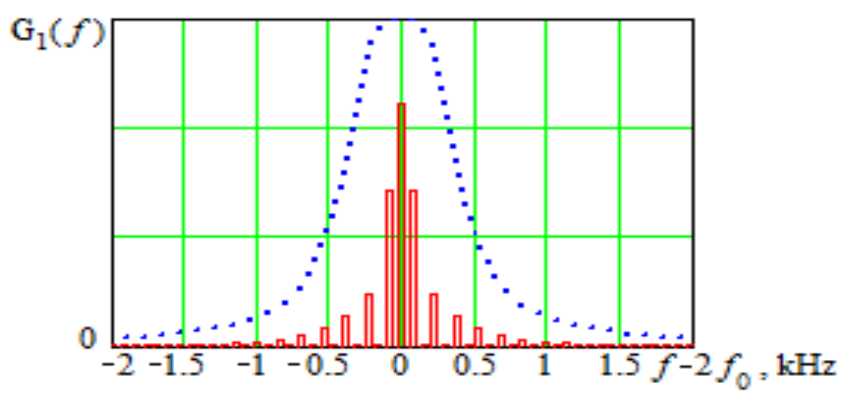

(a)

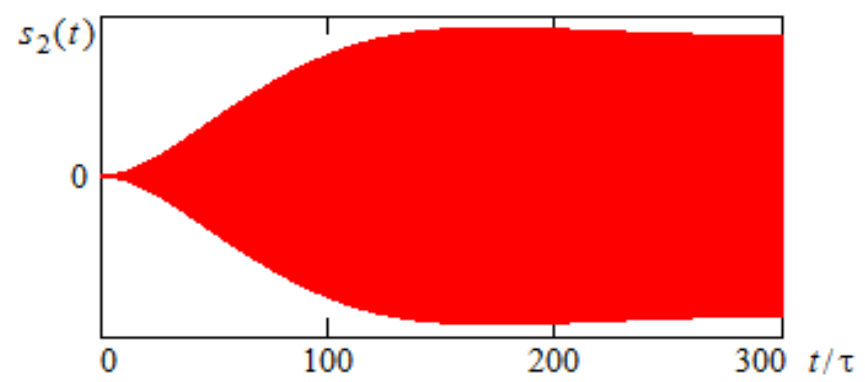

(b)

FIGURE IV. THE SIGNAL AMPLITUDE SPECTRUM AT THE SF INPUT (A) AND THE TIMING WAVEFORM DIAGRAM AT THE SF OUTPUT (B)

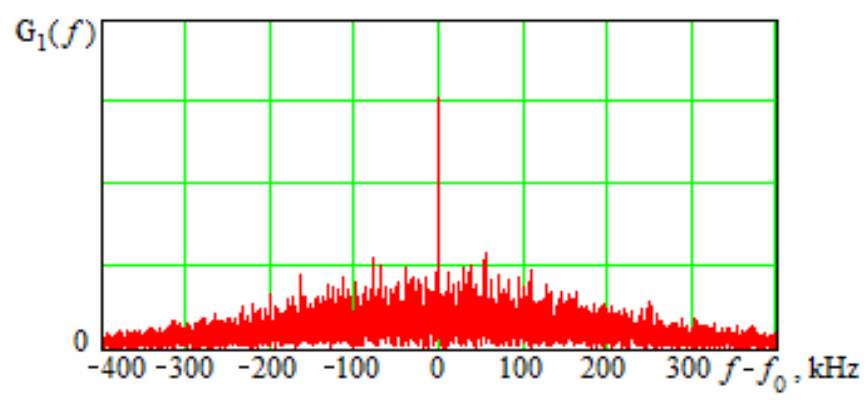

(a)

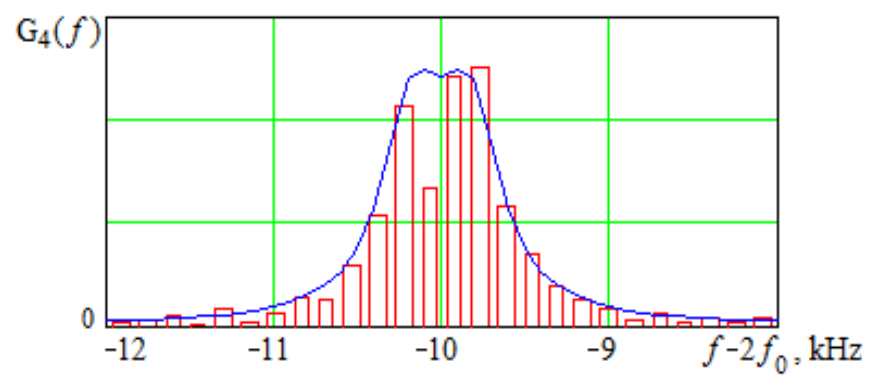

(b)

FIGURE V. THE SIGNAL AMPLITUDE SPECTRUMS AT THE IF INPUT (A) AND OUTPUT (B) 
The SF response $s_{2}(t)$ is passed to the signal detecting device (designated in Figure 1a as SD) which forms the current estimate of the signal component (2) and the SD output signal $s_{3}(t)$ is compared to a threshold in the resolver (designated in Figure 1a as RS) for decision D on signal detection.

In order to generate the threshold formation the interference level at the detector input should be estimated. The spectrum of the output FM signal in the presence of interferences (unlike the similar spectrum, if there are no interferences - see Figure 3) is shown in Figure 5 a. The narrow-band noise filter (designated in Figure 1a as IF) selects the frequency components at a point $2 f_{0}-F_{n}$. Here the value $F_{n}$ is chosen so that the signal spectrum in the specified neighborhood of the frequency $2 f_{0}-F_{n}$ was formed by interferences basically. For example, in Figure $5 \mathrm{~b}$ the spectrum of the IF response for $F_{n}=10 \mathrm{kHz}$ is represented.

The output IF signal is passed to the interference detector (designated in Figure 1 a as ID) including low-frequency filter. The response of this filter sets the threshold level in the resolver.

\section{NOISE IMMUNITY OF THE BINARY PSK SIGNAL DETECTOR}

Let us designate the PSK signal bandwidth matching the input filter $\mathrm{F}$ bandpass as $\Delta f_{s}$, and the narrow-band signal filter bandpass as $\Delta f_{0}$. According to [5], if the interferences can be approximated by the Gaussian white noise with dispersion (within the signal bandwidth) $\sigma_{n \text { in }}^{2}$, then under $\Delta f_{s} \gg \Delta f_{0}$ the process $s_{1}(t)$ at the output of the square-law converter passing through the SF-SD section is normalized and its probability density is described by Gaussian law with good accuracy. In the presence of the PSK signal the mean value $\bar{S}_{3}$ and the dispersion $\sigma_{3}^{2}$ of the output converter response are approximately equal to

$$
\begin{gathered}
\bar{s}_{3}=S^{2} / 2+\sigma_{n \text { in }}^{2}=\sigma_{n \text { in }}^{2}\left(1+h_{0}^{2}\right) \\
\sigma_{3}^{2}=\Delta f_{0} \sigma_{n \text { in }}^{4}\left(1+2 \sqrt{2} h_{0}^{2}\right) / \Delta f_{s} \sqrt{2}=\mu \sigma_{n \text { in }}^{4}\left(1+2 \sqrt{2} h_{0}^{2}\right) / \sqrt{2} .
\end{gathered}
$$

where

$$
h_{0}^{2}=S^{2} / 2 \sigma_{\text {nin }}^{2} .
$$

is the power signal-to-noise ratio at the detector input, and

$$
\mu=\Delta f_{0} / \Delta f_{s}<<1
$$

The one-dimensional probability density of the normalized values $z=s_{3} / \sigma_{\text {min }}$ of the process $s_{3}(t)$ can be approximately written down as

$$
w(y)=\frac{1}{\sqrt{\sqrt{2 \pi} \mu\left(1+2 \sqrt{2} h_{0}^{2}\right)}} \exp \left[-\frac{\left(z-h_{0}^{2}-1\right)^{2}}{\sqrt{2} \mu\left(1+2 \sqrt{2} h_{0}^{2}\right)}\right]
$$

Then, for the case of the normalized threshold $z_{0} \geq 1$, the false-alarm probability $P_{F A}$ is determined by the expression

$$
P_{F A}=1-\frac{1}{\sqrt{\sqrt{2} \pi \mu}} \int_{-\infty}^{z_{0}} \exp \left[-\frac{(z-1)^{2}}{2 \sqrt{2} \mu}\right] d z=\frac{1}{2}\left[1-\operatorname{erf}\left(\frac{z_{0}-1}{\sqrt{\sqrt{2} \mu}}\right)\right]
$$

where $\operatorname{erf}(x)$ is the probability integral (error function) of the

$$
\text { kind of } \operatorname{erf}(x)=2 \int_{0}^{x} \exp \left(-t^{2}\right) d t / \sqrt{\pi} \text {. }
$$

By analogy, the missing probability can be calculated as

$$
\begin{gathered}
P_{M S}=\frac{1}{\sqrt{\sqrt{2} \pi \mu\left(1+2 \sqrt{2} h_{0}^{2}\right)}} \int_{-\infty}^{z_{0}} \exp \left[-\frac{\left(z-h_{0}^{2}-1\right)^{2}}{\sqrt{2} \mu\left(1+2 \sqrt{2} h_{0}^{2}\right)}\right] d z= \\
=\frac{1}{2}\left[1-\operatorname{erf}\left(\frac{h_{0}^{2}+1-z_{0}}{\sqrt{\sqrt{2} \pi \mu\left(1+2 \sqrt{2} h_{0}^{2}\right)}}\right)\right] .
\end{gathered}
$$

In Figure 6 the dependences of the false-alarm probability $P_{F A}$ (curves are on the left) and of the missing probability $P_{M S}$ (curves are on the right) from threshold level is presented for various values of $\mu$ (4) and signal-to-noise ratio $h_{0}^{2}$ (3). As can be seen, the introduced detector possesses a high-noise immunity which is increasing substantially with the signal-tonoise ratio (if $h_{0}^{2} \geq 2$ ) and with decreasing the parameter $\mu$.

The values of the normalized threshold $z_{0}$ lie within limits from 1 to 5 . It means that for the unit transmission factor of SF-SD and NF-ND sections the detector response ${ }^{s_{3}}$ should be compared to the absolute threshold $s_{5}=z_{0} \sigma_{n \text { in }}^{2}$ which lies within limits from $\sigma_{n \text { in }}^{2}$ to $5 \sigma_{n \text { in }}^{2}$. We shall notice, that the optimal normalized threshold $z_{0}$ depends on both $\mu$ and $h_{0}^{2}$. Therefore, for generating ${ }^{z_{0}}$ in the RS, it is necessary to share the IF and SF responses.

If the Neumann-Pirson criterion is used for threshold determination, then the threshold value ${ }^{z_{0}}$ is chosen so as to ensure the specified false-alarm probability level $P_{F A}$. Then, calculating the inverse function to Eq. (5), we obtain the dependences $z_{0}\left(P_{F A}\right)$ shown in Figure 7 for various $\mu$ (4). Apparently, according to the criterion mentioned above, over a large range of false-alarm probability values the threshold is $z_{0}=1 \div 2$ 


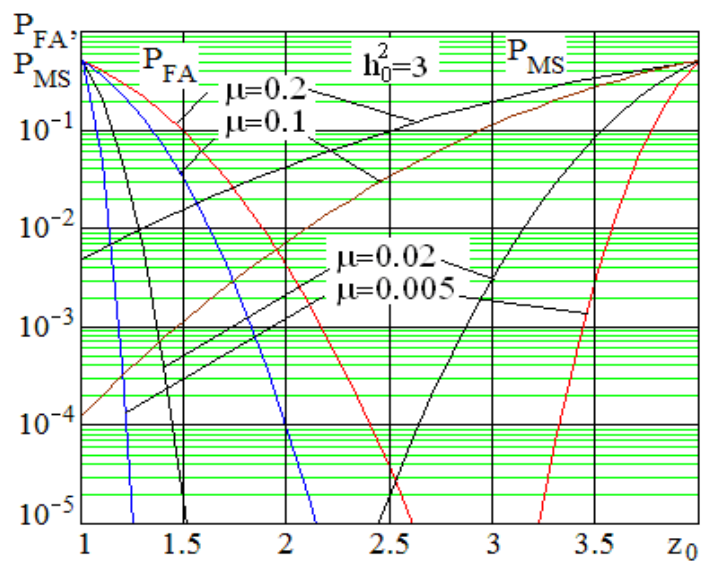

(a)

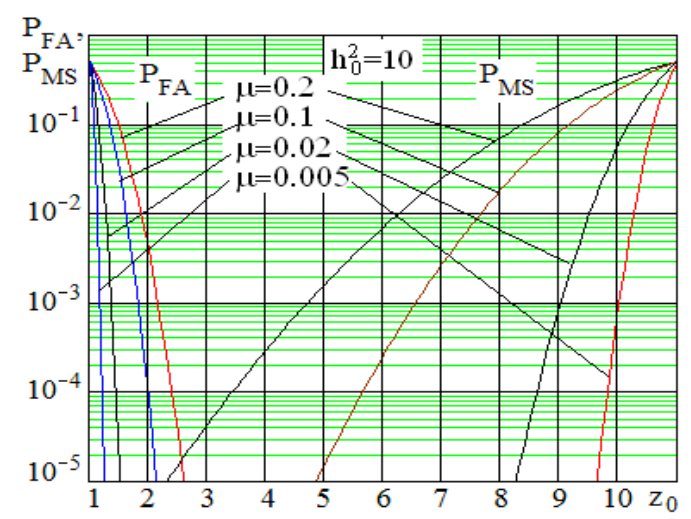

(b)

FIGURE VI. THE FALSE-ALARM AND MISSING PROBABILITY FOR

$$
h_{0}^{2}=3 \text { (A) AND } h_{0}^{2}=10 \text { (B) }
$$

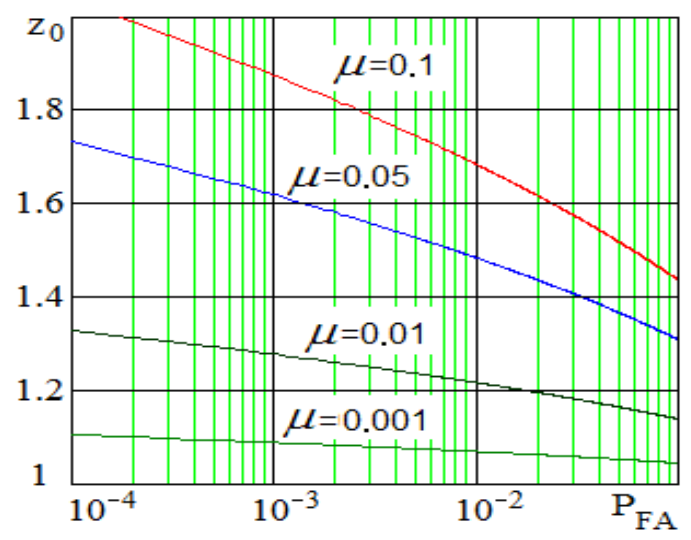

FIGURE VII. THE VALUES OF THRESHOLD ${ }^{z_{0}}$ BY THE NEUMANNPIRSON CRITERION

In Figure 8 by dotted lines the dependences of the falsealarm probability on parameter $\mu$ are drawn for various signalto-noise ratios $h_{0}^{2}$ (3) and $z_{0}=1.3$ or $z_{0}=1.5$. While there the similar dependences of missing probability are plotted by dashed (for $z_{0}=1.3$ ) and solid (for $z_{0}=1.5$ ) lines. As follows from Figure 8, a high-noise immunity of PSK signal detection is provided, if $\mu \leq 0.03$ and $h_{0}^{2} \geq 2$.

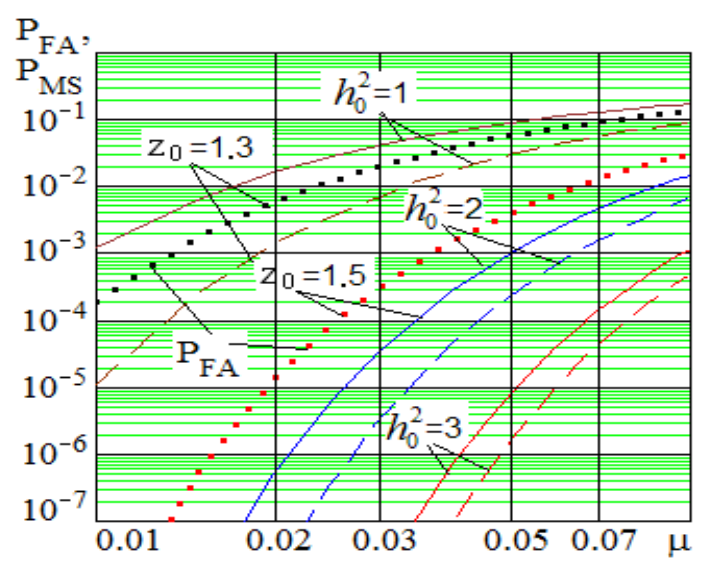

FIGURE VIII. THE FALSE-ALARM AND MISSING PROBABILITIES

So, detecting the PSK signals in a reliable manner can be implemented with small error probabilities and for input signal-to-noise ratios which are not too big. The detection efficiency increases sharply with decreasing the relation of the signal bandwidth to the narrow-band signal filter bandpass. However, in this case the detector response time is also increasing.

\section{CONCLUSION}

The introduced detector provides the possibility of PSK signal extraction with a high-noise immunity, when the signalto-noise ratio is much less than its working value in the receiver highway. It can be simply implemented due to the support by both the analog and digital devices. The efficiency of the considered PSK signal detector is corroborated by statistical simulation.

\section{ACKNOWLEDGMENT}

The reported study was supported by Russian Science Foundation (research project No. 14-49-00079)

\section{REFERENCES}

[1] L.M. Fink, Discrete-message communication theory [in Russian]. Sovetskoe Radio, Moscow, 1970.

[2] J. Proakis, M. Salehi, Digital Communications. McGraw-Hill, New York, 2007.

[3] B. Sklar, Digital Communications: Fundamentals and Applications. Prentice Hall, New Jersey, 2001.

[4] V.P. Litvinenko, Yu.V. Litvinenko, Phase-shift keyed signal detector, Certificate of Authorship № 2527761, Russia, IPC H03D3/00, H04B1/10 (patent application № 2011130806/08, appl. 22.07.2011, publ by Federal Service for Intellectual Property Patents and Trademarks of the Russian Federation 10.09.2014, Bull. 25).

[5] H. Stark, J.W. Woods, Probability and Random Processes with Applications to Signal Processing. Prentice-Hall, New Jersey, 2002. 